\title{
An efficient ordered-alternative test for the randomized blocks design
}

\author{
WILLIAM J. KROSSNER and ALICE FENVESSY, Department of \\ Psychology, Vassar College, Poughkeepsie, N. Y.
}

A table of critical values is presented, a worked example given, and a computer program offered for an asymptotically distribution-free rank test that compares the null hypothesis of no difference in treatments with an alternative hypothesis based on an a priori ordering in treatment effects.

Growing sophistication in psychological theory now often permits an $\mathrm{E}$ to make predictions as to the relative magnitude of the effects of his various treatments in advance of collecting his data and testing them merely for treatment differences. For this increasingly important situation in which the treatment effects can be completely ordered a priori, a rank test proposed by Hollander (1967) would seem to be useful. The test in essence involves the computation of Wilcoxon signed-rank statistics for all pairwise treatment comparisons, the addition of these over pairs, and reference to a critical value.

The procedure for the case of $\mathrm{N}$ blocks and $\mathrm{K}$ treatments, with a null hypothesis of no difference in treatments vs an alternative hypothesis of a decreasing treatment effect from Treatment 1 through Treatment $\mathrm{K}$ is as follows:

(1) Starting at the left, and forming comparisons between Treatments 1 and 2,1 and 3 , through 1 and $K$, and then going on to comparisons between 2 and 3,2 and 4 , and so on, take the differences between the sample value in each block in the first treatment and the sample value in the same block in the second treatment. The result is $K(K-1) / 2$ sets of $N$ differences.

(2) Within each set, disregard zero differences and rank order the remaining ones without regard to sign, assigning Rank 1 to the smallest, up through Rank $\mathbf{N}$ if there are no zero differences. In the case of ties, follow the usual procedure of assigning average ranks.

(3) Within each set, take the sum of ranks of positive differences, i.e., those arising from cases in which the sample value in an earlier treatment is higher than the sample value for a later one. These are the cases that confirm the alternative hypothesis.

(4) Add these rank sums across sets. Compare the total rank sum. $Y$, with the tabulated critical value at significance level alpha, and reject the null hypothesis if $Y$ exceeds the critical value.

This procedure may be illustrated by the analysis of the data of Table 1, which contains reaction times from six of a rather larger number of $S s$ who participated in an unpublished experiment of the senior author which involved the solution of problems expressed in one of four sentence classes. From psycholinguistic considerations, it was predicted that the reaction times for the four classes, presented to Ss in random order, should steadily
Table 1

Reaction Times, sec, for Sentence Analysis

\begin{tabular}{crccc} 
& & \multicolumn{3}{c}{ Sentence Class } \\
Subject & 1 & 2 & 3 & 4 \\
\hline 1 & 5.7 & 3.9 & 3.3 & 3.4 \\
2 & 7.3 & 6.7 & 6.8 & 2.7 \\
3 & 6.6 & 4.4 & 5.2 & 3.1 \\
4 & 6.9 & 7.2 & 6.7 & 3.3 \\
5 & 10.0 & 7.6 & 5.2 & 3.1 \\
6 & 7.9 & 5.1 & 4.8 & 3.7 \\
\hline
\end{tabular}

decrease. These data from a repeated measurements design are to be analyzed as a randomized blocks design in which each $S$ comprises a block. For comments on this approach, see Winer (1962) and Hays (1963).

The six partial sums in order are $20,21,21,15,21$, and 20 , and the total rank sum $Y$ is 118 . From Table $2, C V(6,4,01)$ is 97 , and the null hypothesis is rejected relative to the specified alternative at better than the .01 level.

Table 2 (on the next page) presents critical values rounded to the nearest integer at significance levels of .05 and .01 for $\mathrm{K}$ ranging from 2 through 10 and assorted $N$. Decimal points in the $Y$ rank sum can of course arise only from tied ranks.

Hollander (1967) proved that his test has a high efficiency relative to other rank tests, and his further results would seem to indicate that above a very modest $\mathrm{N}$ and $\mathrm{K}$, the data need not be assumed to be normally distributed for the test to be accurate. Puri and Sen (1968) have recently extended Hollander's work to construct more general classes of ordered-alternative tests.

Inasmuch as the labor of computing ranked differences rises rapidly with increasing $N$ and $K$, an efficient computer routine in either Fortran IV or IBM 360 machine language is available from the senior author to compute the test statistic $Y$ and ascertain significance at levels of $.05, .01$, and .001 .

\section{REFERENCES}

HAYS, W. L. Statistics for psychologists. New York: Holt, Rinehart \& Winston, 1963.

HOLLANDER, M. Rank tests for randomized blocks when the alternatives have an a priori ordering. Annals of Mathematical Statistics, 1967, 38, 867-877.

PURI, M. L., \& SEN, P. K. On Chernoff-Savage tests for ordered altematives in randomized blocks. Annals of Mathematical Statistics, 1968, 39, 967-972.

WINER, B. J. Statistical principles in experimental design. New York: McGraw.Hill, 1962. 
Table 2

Critical Values at the .05 and (.01) Significance Levels

Treatments, $K$

\begin{tabular}{|c|c|c|c|c|c|c|c|c|c|}
\hline locks, N/ & 2 & 3 & 4 & 5 & 6 & 7 & 8 & 9 & 10 \\
\hline 2 & $\begin{array}{l}3 \\
(4)\end{array}$ & (10) & $\begin{array}{l}15 \\
\text { (17) }\end{array}$ & $\begin{array}{c}23 \\
(26)\end{array}$ & $\begin{array}{c}33 \\
(37)\end{array}$ & $\begin{array}{c}44 \\
(50)\end{array}$ & $\begin{array}{c}58 \\
(64)\end{array}$ & $\begin{array}{c}72 \\
(80)\end{array}$ & \\
\hline 3 & $\begin{array}{l}6 \\
(7)\end{array}$ & $\begin{array}{l}15 \\
(18)\end{array}$ & $\begin{array}{l}27 \\
(31)\end{array}$ & $\begin{array}{r}43 \\
(49)\end{array}$ & $\begin{array}{c}62 \\
(69)\end{array}$ & $\begin{array}{l}85 \\
(94)\end{array}$ & $\begin{array}{l}111 \\
(122)\end{array}$ & $\begin{array}{l}140 \\
(153)\end{array}$ & $\begin{array}{r}172 \\
(187\end{array}$ \\
\hline 4 & $\begin{array}{l}10 \\
\text { (11) }\end{array}$ & $\begin{array}{l}24 \\
(28)\end{array}$ & $\begin{array}{l}44 \\
(50)\end{array}$ & $\begin{array}{l}69 \\
(78)\end{array}$ & $\begin{array}{l}101 \\
\text { (111) }\end{array}$ & $\begin{array}{l}137 \\
(151)\end{array}$ & $\begin{array}{r}179 \\
(196)\end{array}$ & $\begin{array}{l}227 \\
(246)\end{array}$ & $\begin{array}{l}280 \\
(303)\end{array}$ \\
\hline 5 & $\begin{array}{l}14 \\
(16)\end{array}$ & $\begin{array}{c}34 \\
(40)\end{array}$ & $\begin{array}{l}64 \\
(72)\end{array}$ & $\begin{array}{l}102 \\
\text { (112) }\end{array}$ & $\begin{array}{l}147 \\
(162)\end{array}$ & $\begin{array}{c}202 \\
(220)\end{array}$ & $\begin{array}{l}264 \\
(286)\end{array}$ & $\begin{array}{c}334 \\
(361)\end{array}$ & $\begin{array}{c}413 \\
(444)\end{array}$ \\
\hline 6 & $\begin{array}{l}18 \\
(22)\end{array}$ & $\begin{array}{c}47 \\
(53)\end{array}$ & $\begin{array}{l}87 \\
(97)\end{array}$ & $\begin{array}{l}139 \\
(153)\end{array}$ & $\begin{array}{c}203 \\
(221)\end{array}$ & $\begin{array}{l}277 \\
(301)\end{array}$ & $\begin{array}{c}364 \\
(392)\end{array}$ & $\begin{array}{c}461 \\
(495)\end{array}$ & $\begin{array}{l}570 \\
(610)\end{array}$ \\
\hline 7 & $\begin{array}{l}24 \\
(28)\end{array}$ & $\begin{array}{l}61 \\
(69)\end{array}$ & $\begin{array}{l}114 \\
(127)\end{array}$ & $\begin{array}{c}183 \\
(200)\end{array}$ & $\begin{array}{r}266 \\
(289)\end{array}$ & $\begin{array}{c}365 \\
(394)\end{array}$ & $\begin{array}{c}479 \\
(515)\end{array}$ & $\begin{array}{c}607 \\
(650)\end{array}$ & $\begin{array}{c}751 \\
(801)\end{array}$ \\
\hline 8 & $\begin{array}{c}30 \\
(35)\end{array}$ & $\begin{array}{c}77 \\
(87)\end{array}$ & $\begin{array}{c}145 \\
(160)\end{array}$ & $\begin{array}{c}232 \\
(253)\end{array}$ & $\begin{array}{c}338 \\
(366)\end{array}$ & $\begin{array}{c}464 \\
(499)\end{array}$ & $\begin{array}{c}609 \\
(652)\end{array}$ & $\begin{array}{c}773 \\
(825)\end{array}$ & $\begin{array}{r}957 \\
(1018)\end{array}$ \\
\hline 9 & $\begin{array}{c}36 \\
(42)\end{array}$ & $\begin{array}{r}95 \\
(106)\end{array}$ & $\begin{array}{l}178 \\
(196)\end{array}$ & $\begin{array}{c}286 \\
(311)\end{array}$ & $\begin{array}{c}418 \\
(451)\end{array}$ & $\begin{array}{l}574 \\
(616)\end{array}$ & $\begin{array}{c}754 \\
(806)\end{array}$ & $\begin{array}{c}958 \\
(1020)\end{array}$ & $\begin{array}{l}1186 \\
(1258)\end{array}$ \\
\hline 10 & $\begin{array}{c}44 \\
(50)\end{array}$ & $\begin{array}{l}114 \\
(128)\end{array}$ & $\begin{array}{r}215 \\
(236)\end{array}$ & $\begin{array}{c}346 \\
(376)\end{array}$ & $\begin{array}{c}506 \\
(545)\end{array}$ & $\begin{array}{l}696 \\
(745)\end{array}$ & $\begin{array}{c}915 \\
(975)\end{array}$ & $\begin{array}{c}1163 \\
(1235)\end{array}$ & $\begin{array}{l}1440 \\
(1524)\end{array}$ \\
\hline 11 & $\begin{array}{l}52 \\
(59)\end{array}$ & $\begin{array}{l}136 \\
(151)\end{array}$ & $\begin{array}{c}256 \\
(280)\end{array}$ & $\begin{array}{c}412 \\
(445)\end{array}$ & $\begin{array}{c}603 \\
(647)\end{array}$ & $\begin{array}{c}829 \\
(885)\end{array}$ & $\begin{array}{c}1090 \\
(1159)\end{array}$ & $\begin{array}{l}1387 \\
(1469)\end{array}$ & $\begin{array}{c}1718 \\
(1814)\end{array}$ \\
\hline 12 & $\begin{array}{l}60 \\
(69)\end{array}$ & $\begin{array}{l}159 \\
(176)\end{array}$ & $\begin{array}{c}300 \\
(327)\end{array}$ & $\begin{array}{c}483 \\
(521)\end{array}$ & $\begin{array}{c}707 \\
(758)\end{array}$ & $\begin{array}{r}973 \\
(1037)\end{array}$ & $\begin{array}{l}1281 \\
(1359)\end{array}$ & $\begin{array}{l}1630 \\
(1723)\end{array}$ & $\begin{array}{r}2019 \\
(2129)\end{array}$ \\
\hline 13 & $\begin{array}{c}69 \\
(79)\end{array}$ & $\begin{array}{c}183 \\
(203)\end{array}$ & $\begin{array}{c}347 \\
(377)\end{array}$ & $\begin{array}{c}559 \\
(602)\end{array}$ & $\begin{array}{c}820 \\
(877)\end{array}$ & $\begin{array}{c}1129 \\
(1201)\end{array}$ & $\begin{array}{c}1486 \\
(1574)\end{array}$ & $\begin{array}{c}1891 \\
(1996)\end{array}$ & $\begin{array}{c}2344 \\
(2468)\end{array}$ \\
\hline 14 & $\begin{array}{c}79 \\
(90)\end{array}$ & $\begin{array}{c}210 \\
(231)\end{array}$ & $\begin{array}{l}397 \\
(431)\end{array}$ & $\begin{array}{c}641 \\
(689)\end{array}$ & $\begin{array}{c}941 \\
(1004)\end{array}$ & $\begin{array}{l}1296 \\
(1376)\end{array}$ & $\begin{array}{l}1707 \\
(1804)\end{array}$ & $\begin{array}{c}2172 \\
(2290)\end{array}$ & $\begin{array}{c}2694 \\
(2831)\end{array}$ \\
\hline 15 & $\begin{array}{c}89 \\
(101)\end{array}$ & $\begin{array}{c}238 \\
(261)\end{array}$ & $\begin{array}{c}451 \\
(488)\end{array}$ & $\begin{array}{c}728 \\
(781)\end{array}$ & $\begin{array}{l}1069 \\
(1139)\end{array}$ & $\begin{array}{c}1474 \\
(1562)\end{array}$ & $\begin{array}{c}1942 \\
(2050)\end{array}$ & $\begin{array}{c}2473 \\
(2602)\end{array}$ & $\begin{array}{r}3066^{\circ} \\
(3218)\end{array}$ \\
\hline 20 & $\begin{array}{c}149 \\
(167)\end{array}$ & $\begin{array}{c}403 \\
(439)\end{array}$ & $\begin{array}{c}768 \\
(826)\end{array}$ & $\begin{array}{c}1245 \\
(1326)\end{array}$ & $\begin{array}{c}1833 \\
(1940)\end{array}$ & $\begin{array}{c}2531 \\
(2667)\end{array}$ & $\begin{array}{c}3339 \\
(3505)\end{array}$ & $\begin{array}{c}4257 \\
(4455)\end{array}$ & $\begin{array}{c}5284 \\
(5516)\end{array}$ \\
\hline 25 & $\begin{array}{c}224 \\
(250)\end{array}$ & $\begin{array}{c}609 \\
(660)\end{array}$ & $\begin{array}{c}1167 \\
(1247)\end{array}$ & $\begin{array}{c}1897 \\
(2009)\end{array}$ & $\begin{array}{c}2796 \\
(2945)\end{array}$ & $\begin{array}{c}3866 \\
(4054)\end{array}$ & $\begin{array}{c}5105 \\
(5336)\end{array}$ & $\begin{array}{c}6514 \\
(6788)\end{array}$ & $\begin{array}{r}8090 \\
(8413)\end{array}$ \\
\hline 30 & $\begin{array}{l}312 \\
(346)\end{array}$ & $\begin{array}{c}857 \\
(923)\end{array}$ & $\begin{array}{l}1647 \\
(1751)\end{array}$ & $\begin{array}{c}2681 \\
(2828)\end{array}$ & $\begin{array}{c}3958 \\
(4152)\end{array}$ & $\begin{array}{c}5477 \\
(5723)\end{array}$ & $\begin{array}{c}7238 \\
(7539)\end{array}$ & $\begin{array}{c}9239 \\
(9599)\end{array}$ & $\begin{array}{l}11482 \\
(11904)\end{array}$ \\
\hline 35 & $\begin{array}{c}415 \\
(457)\end{array}$ & $\begin{array}{l}1145 \\
(1228)\end{array}$ & $\begin{array}{c}2206 \\
(2337)\end{array}$ & $\begin{array}{c}3597 \\
(3782)\end{array}$ & $\begin{array}{c}5316 \\
(5561)\end{array}$ & $\begin{array}{c}7362 \\
(7672)\end{array}$ & $\begin{array}{r}9735 \\
(10114)\end{array}$ & $\begin{array}{l}12433 \\
(12886)\end{array}$ & $\begin{array}{c}15456 \\
(15987)\end{array}$ \\
\hline 40 & $\begin{array}{c}532 \\
(583)\end{array}$ & $\begin{array}{l}1474 \\
(1575)\end{array}$ & $\begin{array}{c}2846 \\
(3006)\end{array}$ & $\begin{array}{c}4645 \\
(4871)\end{array}$ & $\begin{array}{c}6871 \\
(7169)\end{array}$ & $\begin{array}{c}9521 \\
(9899)\end{array}$ & $\begin{array}{l}12595 \\
(13058)\end{array}$ & $\begin{array}{l}16093 \\
(16645)\end{array}$ & $\begin{array}{c}20012 \\
(20660)\end{array}$ \\
\hline 45 & $\begin{array}{c}663 \\
(724)\end{array}$ & $\begin{array}{c}1843 \\
(1964)\end{array}$ & $\begin{array}{c}3564 \\
(3755)\end{array}$ & $\begin{array}{c}5824 \\
(6093)\end{array}$ & $\begin{array}{c}8621 \\
(8977)\end{array}$ & $\begin{array}{l}11953 \\
(12403)\end{array}$ & $\begin{array}{c}15819 \\
(16370)\end{array}$ & $\begin{array}{c}20218 \\
(20876)\end{array}$ & $\begin{array}{c}25149 \\
(25921)\end{array}$ \\
\hline 50 & $\begin{array}{c}808 \\
\text { (878) }\end{array}$ & $\begin{array}{c}2253 \\
(2394)\end{array}$ & $\begin{array}{c}4362 \\
(4585)\end{array}$ & $\begin{array}{c}7134 \\
(7449)\end{array}$ & $\begin{array}{c}10567 \\
(10983)\end{array}$ & $\begin{array}{l}14657 \\
(15183)\end{array}$ & $\begin{array}{c}19405 \\
(20049)\end{array}$ & $\begin{array}{c}24808 \\
(25578)\end{array}$ & $\begin{array}{r}3086 \\
(3176\end{array}$ \\
\hline
\end{tabular}

